\title{
microRNA for determining the age-related myogenic capabilities of skeletal muscle
}

\author{
Kwang-Pyo Lee ${ }^{1, \#}$, Yeo Jin Shin ${ }^{1,2, \#} \mathcal{E}$ Ki-Sun Kwon ${ }^{1,2, *}$ \\ ${ }^{1}$ Aging Research Institute, Korea Research Institute of Bioscience and Biotechnology, Daejeon 34141 , ${ }^{2}$ Department of Functional \\ Genomics, Korea University of Science and Technology, Daejeon 34113, Korea
}

Skeletal muscle exhibits a loss of muscle mass and function with age. Decreased regenerative potential of muscle stem/ progenitor cells is a major underlying cause of sarcopenia. We analyzed microRNAs (miRNA) that are differentially expressed in young and old myoblasts, to identify novel intrinsic factors that play a degenerative role in aged skeletal muscle. miR-431, one of decreasing miRNAs in old myoblasts, improved the myogenic differentiation when overexpressed in old myoblast, but suppressed their myogenic capability in knockdowned young myoblasts. We found that miR-431 directly binds to $3{ }^{`}$ untranslated regions (UTR) of Smad4 mRNA, and decreases its expression. Given that SMAD4 is one of the downstream effectors of TGF- $\beta$, a well-known degenerative signaling pathway in myogenesis, the decreased miR-431 in old myoblast causes SMAD4 elevation, thus resulting in defective myogenesis. Exogenous expression of miR-431 greatly improved the muscle regeneration in the cardiotoxin-injured hindlimb muscle of old mice by reducing SMAD4 levels. Since the miR-431 seed sequence is conserved in human SMAD4 3'UTR, miR-431 regulates the myogenic capacity of human skeletal myoblasts in the same manner. Our results suggest that age-associated miR-431 is required for the maintenance of the myogenic capability in myoblasts, thus underscoring its potential as a therapeutic target to slow down muscle aging. [BMB Reports 2015; 48(11): 595-596]

Age-related loss of muscle mass and function can be triggered by various factors such as hormonal imbalances, inflammation,

*Corresponding author. E-mail: kwonks@kribb.re.kr

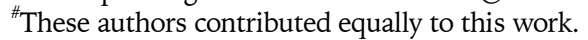

http://dx.doi.org/10.5483/BMBRep.2015.48.11.211

Received 16 October 2015

Keywords: Differentiation, miR-431, Muscle aging, Myoblast, SMAD4

Abbreviations: miRNA, microRNA; SMAD4, SMAD family member 4 ; TGF- $\beta$, transforming growth factor $\beta$; PAX7, paired box gene 7 ; MYOD, myogenic differentiation 1

Perspective to: Lee et al (2015), miR-431 promotes differentiation and regeneration of old skeletal muscle by targeting Smad4, Genes Dev, 29(15):1605-17, doi: 10.1101/gad.263574.115. motor neuron degeneration, and oxidative stress. In addition, the number of adult muscle stem/progenitor cells, known as satellite cells, gradually decreases and their myogenic capability declines with age. Satellite cells ( $\mathrm{PAX} 7^{+} \mathrm{MYOD}^{-}$) in old muscle tissues showed age-related phenotypes, such as loss of self-renewal and intrinsic regenerative capacities, thus undergoing a senescent state. Differentiation into myotubes requires activation of satellite cells, termed myoblast $\left(\mathrm{PAX}^{+} \mathrm{MYOD}^{+}\right)$. Myoblasts in turn differentiate into myotubes or proliferate themselves. However, after activation of satellite cells into myoblasts, the mechanisms responsible for the decreased myogenic capacity of aged myoblasts has remained unclear.

To find miRNAs differentially expressed in myoblasts with age, we analyzed miRNA expression profiles in primary myoblasts isolated from hind limb muscles of 3-month-old (hereafter designated 'young myoblasts') and 27-month-old (hereafter designated 'old myoblasts') using next-generation sequencing. We identified 118 mature miRNAs differentially expressed between young and old myoblasts. 47 miRNAs were significantly upregulated, and 71 miRNAs were down-regulated in old myoblasts. Interestingly, we found that 63 of the 71 miRNAs (89\%) reduced in old myoblasts were located in Dlk-Dio3 genomic region of chromosome 12 . We have recently reported that most miRNAs decreased in aged muscle tissues were also located in the same genomic locus, suggesting that miRNAs expressed in this genomic region might play an important role in muscle aging process.

Among the miRNAs expressed from Dlk-Dio3 genomic region, we focused on miR-431 which maximally improved the myogenic capability of old myoblasts after transfection of its miRNA-mimic. Using bioinformatic and biochemical analyses, we found that miR-431 directly interacts with 3'UTR of Smad4 mRNA. Consequently, SMAD4 levels were decreased in miR431 transfected old myoblasts or $\mathrm{C} 2 \mathrm{C} 12$ cells. Interestingly, SMAD4 protein levels were also upregulated in cardiotoxin-injured old muscle tissues, wherein the expression levels of miR-431 were downregulated.

Transforming growth factor $\beta$ (TGF- $\beta$ ) signaling is known to represses myogenesis by decreasing the transcriptional activity of myogenic regulatory factors. Moreover, TGF- $\beta$ signaling also inhibits aged muscle regeneration. Old muscle generates high levels of TGF- $\beta$, which in turn activates TGF- $\beta$ receptors. 

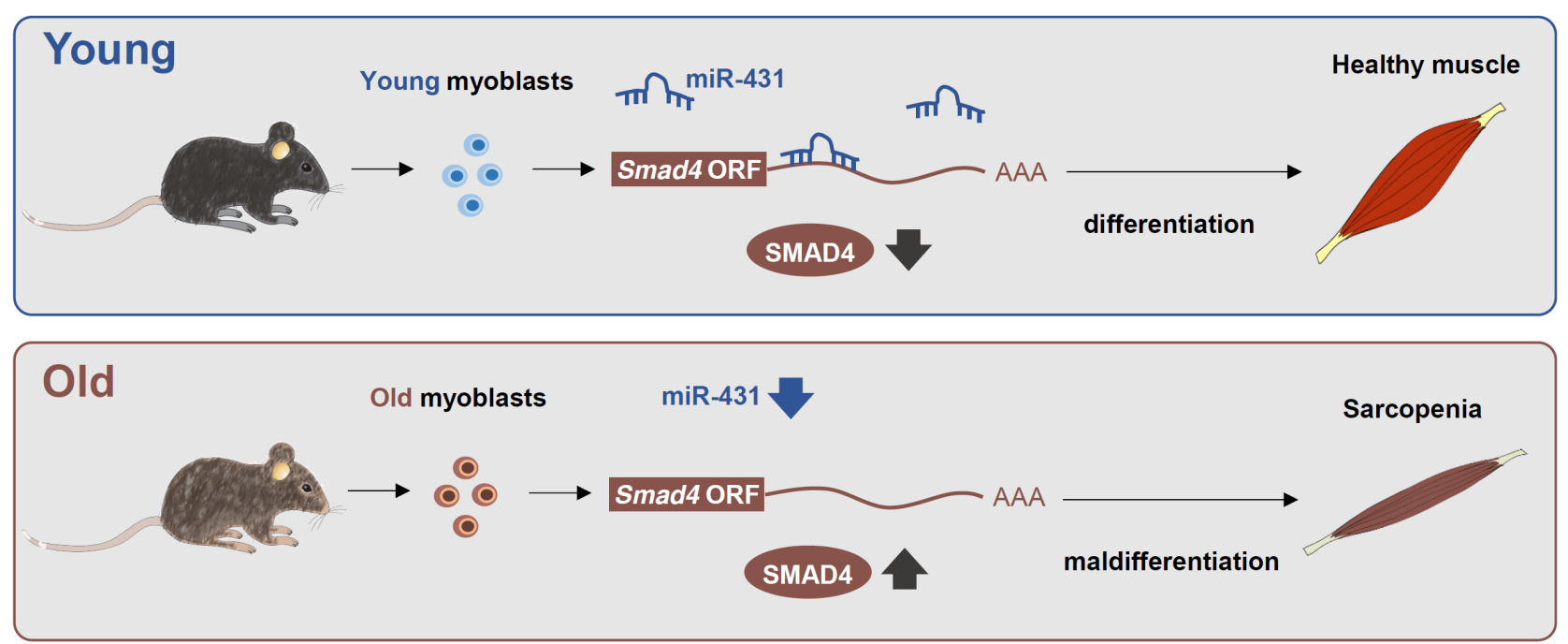

Fig. 1. Proposed model for the regulation of myoblast differentiation by miR-431 in different ages. Down-regulated miR-431 in old $\mathrm{PAX}^{+} \mathrm{MYOD}^{+}$myoblasts results in increased levels of SMAD4, one of the downstream effectors of TGF- $\beta$ signaling, thereby interfering with their normal myogenic capability.

Thereafter, the phosphorylated SMAD2/3 forms a heterotrimeric complex with SMAD4, the common mediator SMAD. This SMAD complex translocates into the nucleus and turns on the target genes that inhibit regenerative capacity of muscle stem/progenitor cells. We thus checked whether miR-431 could control TGF- $\beta$ signaling. Overexpression of miR-431 ameliorated the delay of myogenesis seen in recombinant TGF- $\beta$ treated myoblasts. Moreover, miR-431 suppressed the increased luciferase activity of $4 \mathrm{xSBE}-\mathrm{luc}$ vector, containing four SMAD binding elements in its promoter region, in $\mathrm{C} 2 \mathrm{C} 12$ cells treated with TGF- $\beta$, Activin A or Myostatin.

Our results demonstrated that ectopic miR-431 injection restores the muscle regeneration of old mice and reduced SMAD4 levels. The newly formed myofibers were significantly larger in both miR-431 or siSmad4 injected muscle tissues than those in control muscle tissues, suggesting the idea that SMAD4 might be a promising therapeutic target for healthy muscle aging. Since the mouse miR-431 seed sequence in the Smad4 3 'UTR is conserved in the human SMAD4 3 'UTR, antagonization of miR-431 robustly repressed myogenic differ- entiation of human skeletal muscle myoblasts. Consistent with mouse data, SMAD4 protein levels were lower in human myoblasts transfected with the miR-431 mimic than those in control cells.

In conclusion, our results suggest that miR-431 is required for maintenance of the muscle differentiation of $\mathrm{PAX} 7^{+} \mathrm{MYOD}^{+}$ myoblasts by targeting Smad4 (Fig. 1), thereby promoting regeneration in old mouse skeletal muscle. We also propose that miR-431 may be a valuable target for development of therapeutics to slow down muscle aging.

\section{ACKNOWLEDGEMENTS}

This work was supported by grants from the Bio \& Medical Technology Development Program (20110030133 and 2013M3A9B6076413, K.-S.K.) of the National Research Foundation (NRF), which is funded by the Ministry of Science, ICT \& Future Planning (MSIP), and the KRIBB Research Initiative Program. 